\title{
Risk estimation in autosomal dominant disorders with reduced penetrance
}

\author{
ALAN E H EMERY \\ From The Medical School, University of Edinburgh, Edinburgh EH8 $9 A G$.
}

SUMMARY The occurrence in a family of an isolated case of an autosomal dominant disorder with reduced penetrance presents a difficult problem in genetic counselling. It is shown that in such a situation the risk of recurrence in subsequent offspring is given by:

$$
\frac{P(1-P)}{2\left(1-P f^{\prime}\right)}
$$

where $P$ is the penetrance $(0<P<1)$ and $\mathrm{f}^{\prime}$ the relative fitness $\left(0<\mathrm{f}^{\prime}<1\right)$ of affected individuals. In all cases the risks of recurrence will exceed 1 in 20 unless penetrance is high (greater than 0.90 ) and the relative fitness of affected persons is low (less than $0 \cdot 6$ ).

Genetic counselling in a family with an isolated case of an autosomal dominant disorder is relatively straightforward if the disorder in question is always fully penetrant, as in achondroplasia. In this situation, barring illegitimacy, the affected subject must be a new mutation and therefore the chance of recurrence in any subsequent child is negligible. However, in disorders with reduced penetrance, an isolated case in a family may not be a new mutation because one of the parents may be a clinically unaffected heterozygote. Pauli and Motulsky ${ }^{1}$ derived equations for determining the risks of recurrence in familial cases of disorders with reduced penetrance, but did not consider the problem of the isolated case. Aylsworth and Kirkman ${ }^{2}$ studied the problem, but related the risks to mutation rates which are notoriously difficult to assess with any accuracy, especially when there is reduced penetrance. More recently, Friedman ${ }^{3}$ examined the problem and used fitness as a means of estimating mutation. However, he considered fitness regardless of phenotype, but this is not appropriate because in practice, in disorders with reduced penetrance, fitness is only assessed for affected heterozygotes. Here, a formula is derived which takes into account fitness of affected heterozygotes. It is then shown that the risks of recurrence in offspring born after an isolated case will always be greater than 1 in 20 unless penetrance exceeds 0.90 and fitness is less than $0 \cdot 6$.

\section{Derivation}

The derivation is based on Bayesian methods. In the situation under consideration the prior probability that both parents are heterozygous can be ignored, since we are dealing with rare disorders. The probability that either parent is heterozygous, but appears normal because the gene is non-penetrant, is $4 p q(1-P)$ where $p$ and $q$ are the frequencies of the mutant gene and its normal allele and $P$ is the penetrance. Given that one of the parents is heterozygous, then the conditional probability of their having a child who has inherited the mutant gene and is also affected is $P / 2$. On the other hand, given that neither parent is heterozygous, then the conditional probability of their having a child with a new dominant mutation is $2 \mu$, which is equal to $2 p q(1-f)$ where $f$ is the relative biological fitness, and assuming there is balance between mutation and selection. The probability of an affected child is then $2 p q(1-f) P$. The joint and posterior probabilities are then calculated in the usual manner.

$\begin{array}{lll}\text { Probability } & \begin{array}{l}\text { Either parent } \\ \text { heterozygous but } \\ \text { appears normal }\end{array} & \begin{array}{l}\text { Neither parent } \\ \text { heterozygous }\end{array} \\ \text { Prior } & 4 \mathrm{pq}(1-\mathrm{P}) & \sim 1 \\ \text { Conditional } & \mathrm{P} / 2 & 2 \mathrm{pq}(1-\mathrm{f}) \mathrm{P} \\ \text { Joint } & 2 \mathrm{pq}(1-\mathrm{P}) \mathrm{P} & 2 \mathrm{pq}(1-\mathrm{f}) \mathrm{P}\end{array}$

The posterior probability that one of the parents is heterozygous is therefore: 


$$
\begin{gathered}
\frac{2 \mathrm{pq}(1-\mathrm{P}) \mathrm{P}}{2 \mathrm{pq}(1-\mathrm{P}) \mathrm{P}+2 \mathrm{pq}(1-\mathrm{f}) \mathrm{P}} \\
=\quad \frac{1-\mathrm{P}}{2-\mathrm{P}-\mathrm{f}}
\end{gathered}
$$

The risk of the next child inheriting the abnormal gene and also being affected is therefore:

$$
\frac{P(1-P)}{2(2-P-f)}
$$

However, since in practice fitness is assessed only for affected heterozygotes (say $\mathrm{f}^{\prime}$ ) in disorders with reduced penetrance, the fitness of all heterozygotes (f) will be somewhat greater. Using fitness values derived from affected subjects only in equation (2) will therefore tend to underestimate risks calculated in this way.

Since selection against all heterozygotes (s) is equal to selection against those that are affected $\left(s^{\prime}\right)$ multiplied by penetrance:

$$
\text { therefore } \begin{aligned}
\mathbf{s} & =\mathrm{s}^{\prime} \mathrm{P} \\
(1-\mathbf{f}) & =\left(1-\mathbf{f}^{\prime}\right) \mathrm{P} \\
\mathbf{f} & =1-\left(1-\mathbf{f}^{\prime}\right) \mathrm{P}
\end{aligned}
$$

Substituting this value of $f$ in equation (2), the risk to the next child becomes:

$$
\frac{\mathrm{P}(1-\mathrm{P})}{2\left\{2-\mathrm{P}-\left[1-\left(1-\mathrm{f}^{\prime}\right) \mathrm{P}\right]\right\}}
$$

which reduces to:

$$
\frac{P(1-P)}{2\left(1-P f^{\prime}\right)}
$$

If there is no reduction in fitness the risk simply becomes $\mathrm{P} / 2$.

TABLE Risks of recurrence in offspring born after an isolated case of a disorder with reduced penetrance $(P)$ and fitness $\left(\bar{f}^{\prime}\right)$.

\begin{tabular}{llllllll}
\hline Penetrance & \multicolumn{7}{l}{ Fitness } \\
\cline { 2 - 7 } & 0.3 & 0.4 & 0.5 & 0.6 & 0.7 & 0.8 & 0.9 \\
\hline 0.95 & 0.033 & 0.038 & 0.045 & 0.055 & 0.071 & 0.099 & 0.164 \\
0.93 & 0.045 & 0.052 & 0.061 & 0.074 & 0.093 & 0.127 & 0.200 \\
0.90 & 0.062 & 0.070 & 0.082 & 0.098 & 0.122 & 0.161 & 0.237 \\
0.80 & 0.105 & 0.118 & 0.133 & 0.154 & 0.182 & 0.222 & 0.286 \\
0.70 & 0.133 & 0.146 & 0.162 & 0.181 & 0.206 & 0.239 & 0.284 \\
0.60 & 0.146 & 0.158 & 0.171 & 0.187 & 0.207 & 0.231 & 0.261 \\
\hline
\end{tabular}

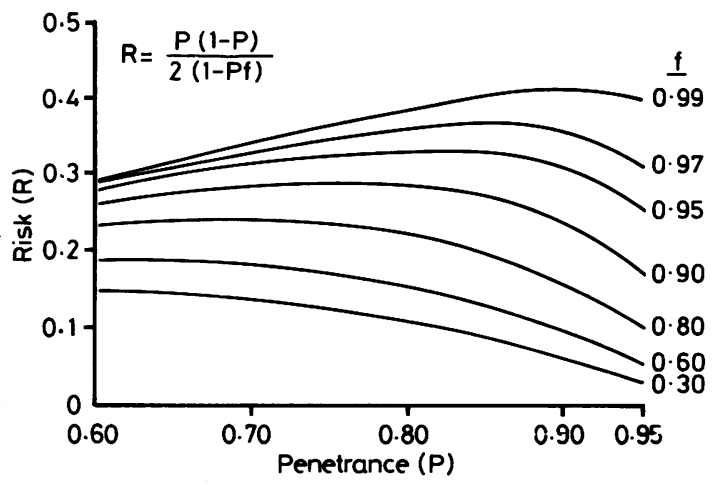

FIGURE Risks of recurrence in offspring born after an isolated case of a disorder with reduced penetrance and fitness.

Risks derived in this way for various values of $\mathbf{P}$ and $f^{\prime}$ are given in the table. However, they only apply to disorders present at birth or when the parents exceed the age at which constant penetrance is reached, and do not apply to disorders where onset is in later life, such as polyposis coli or Huntington's chorea.

\section{Discussion}

For those disorders which confer sterility or in which affected subjects die before adulthood $\left(f^{\prime}=0\right)$, then the value of the risk equation (4) maximises at $12 \cdot 5 \%$, equivalent to a penetrance of $0 \cdot 50$. Only if penetrance exceeds $0 \cdot 88$ (or less than 0.12 but which then questions the actual mode of inheritance) does the risk become less than 1 in 20 . A risk of 1 in 20 or less is generally accepted as low and more likely to be accepted by most couples.

For the majority of autosomal dominant disorders with reduced penetrance fitness, however, is not zero. From the table a recurrence risk of 1 in 20 or less is only reached if penetrance exceeds 0.90 and fitness is less than 0.6 (as seems likely, for example, in tuberous sclerosis ${ }^{4}$ ). The risks of recurrence are particularly influenced by estimates of fitness when penetrance is high (figure). An accurate assessment of fitness is therefore important, but unfortunately this information is only available in a few disorders. Further, estimates of penetrance depend on the thoroughness with which family members have been investigated and the ages of those studied. Disorders in which data are available and can be used to illustrate the application of the method include tuberous sclerosis and myotonic dystrophy, for example. In tuberous sclerosis ${ }^{4}$ penetrance is at 
least 0.95 and fitness is about 0.30 , and therefore the risk of recurrence after an isolated case is $3.3 \%$. In myotonic dystrophy, ${ }^{5}$ by early adulthood penetrance is at least 0.95 and fitness is about 0.75 , so the risk of recurrence after an isolated case is $8.3 \%$.

As techniques for detecting heterozygotes become increasingly more sophisticated and sensitive, so the proportion of heterozygotes who remain completely undetected may become so small that penetrance is virtually complete. In the situation where both parents can be shown not to be heterozygotes, irrespective of the fitness of affected persons, there would be virtually no risk to subsequent children.

\section{References}

' Pauli RM, Motulsky AG. Risk counselling in autosomal dominant disorders with undetermined penetrance. $J$ Med Genet $1981 ; 18: 340-3$.

2 Aylsworth AS, Kirkman HN. Genetic counselling for autosomal dominant disorders with incomplete penetrance. Birth Defects $1979 ; \mathbf{X V}(5 \mathrm{C}): 25-38$.

${ }^{3}$ Friedman JM. Genetic counselling for autosomal dominant diseases with a negative family history. Clin Genet 1985;27: 68-71.

${ }^{4}$ Nevin NC, Pearce WG. Diagnostic and genetic aspects of tuberous sclerosis. J Med Genet 1968;5:273-80.

5 Harper PS. Myotonic dystrophy. London: Saunders, 1979:229.

Correspondence and requests for reprints to Professor A E H Emery, Medical School, University of Edinburgh, Teviot Place, Edinburgh EH8 9AG. 